\title{
EBSD - a Powerful Tool for the Analysis of Magnetic Materials
}

\author{
Dominic Hohs, Tvrtko Grubesa, David Schuller, Timo Bernthaler, Dagmar Goll and Gerhard Schneider
}

\section{Aalen University, Materials Research Institute, Aalen, Germany}

Tailored hard and soft magnetic materials play a key role for efficient next generation electrical energy converters. Besides the chemical composition, the microstructure design is essential for high performance magnets. Hard magnetic materials feature a high coercive field and high remanence, which requires small grain sizes and a high degree of alignment, respectively. Both properties depend on the manufacturing process of hard magnetic materials, which involves the alignment of hard magnetic powder in an external magnetic field before compaction. By means of liquid-phase sintering a microstructure consisting of hard magnetic anisotropic aligned grains is obtained. The grains are magnetically isolated from each other by a non-magnetic grain boundary phase [1]. In contrast, soft magnets like electrical steels are characterized by a low coercive field. This allows high frequency applications with low energy losses. The optimal grain size of electrical steels for automotive applications is between $100-150 \mu \mathrm{m}$ and is achieved due to a combination of rolling and annealing steps [2]. For the final geometry of the motor components mechanical punching processes are required. Therefore, elastic strains and plastic deformation remain in the structure. The design of the structure of hard and soft magnetic materials represents one of the major challenges to realize improved magnetic properties for tailored electromobile applications.

Scanning electron microscopy (SEM) in combination with electron backscatter diffraction (EBSD) is a powerful tool (here: Zeiss Sigma $300 \mathrm{VP}$, EDAX) to analyze the microstructure of both, hard and soft magnetic materials [3]. At crystal lattice planes, the electron beam is diffusely scattered in all directions. Therefore, numerous electrons fulfill Bragg's law. Each crystal type and orientation generates a specific diffraction pattern. Using the inverse pole figure (IPF), the local orientation of each measurement point is indicated. On one hand, measurement points with the same orientation can be related to a grain, which allows the individual determination of size and alignment of all the grains visible at the sample surface. For example the degree of alignment of the field oriented Fe-Nd-B magnets or the rolling texture of electrical steels can be examined in detail. On the other hand, if the misorientation within a grain is evaluated, plastic deformations can be quantified [4]. The reference orientation deviation (ROD) reveals the highest misorientation in comparison with a certain reference or an average orientation within a grain. The kernel average misorientation (KAM) compares neighboring measurement points to their orientation and highlights finest local deviations. As a result, the influence of fabrication process of cutting the electrical steels and the caused plastic deformation at the contact region can be made visible by these two tools. In addition, crystal lattice defects like high displacement densities or grain boundaries lead to diffuse diffraction lines which can be described in terms of the pattern contrast or the image quality (IQ). The potential of EBSD to analyze magnetic materials relating to grain size distribution, grain orientation and plastic deformation will be presented in more detail in this contribution.

Some examples of obtained results are given in the following. Figure 1 shows the grain size distribution and the degree of alignment of three Fe-Nd-B magnets produced by axial, transversal and isostatic pressing. A correlation between degree of alignment and remanence can be observed. The isostatically pressed magnet reaches a higher degree of alignment (97\%), leading to a higher remanence (1.42 T) 
compared to the axially pressed magnet $(<92 \%, 1.31 \mathrm{~T})$. Figure 2 deals with investigations of the plastic deformations at the cutting edges of electrical steels. They are revealed by the reference orientation deviation (ROD) method (in Figure 2, the red color indicates orientation deviations of $10^{\circ}$ or more to the reference orientation of each grain). In addition, the quality of the diffraction pattern (IQ) decreases at deformed parts of the grains due to the high displacement density.

\section{References:}

[1] D. Goll et al., Naturwissenschaften 87 (2000), p. 423.

[2] F.J.G. Landgraf et al., Journal of Magnetism and Magnetic Materials 323 (2011), p. 2335.

[3] S.I. Wright et al., Microscopy and Microanalysis 17 (2011), p. 316.

[4] L.N. Brewer et al., Microscopy and Microanalysis 12 (2006), p. 85.
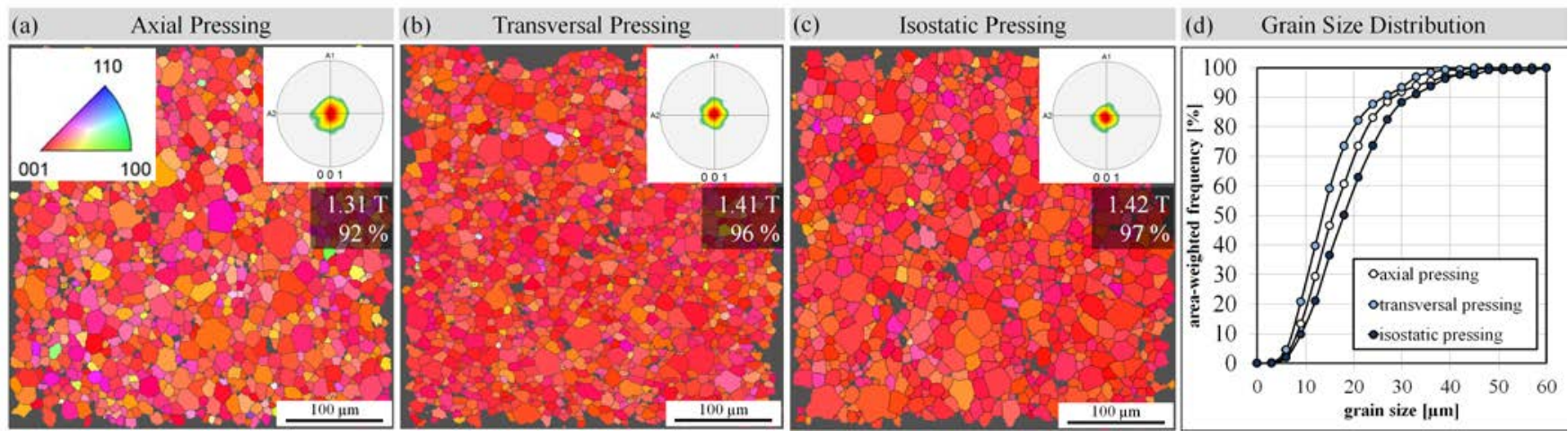

Figure 1. Fe-Nd-B magnets with different degrees of alignment and resulting remanence (a) axial pressing 92\%, $1.31 \mathrm{~T}$ (b) transversal pressing 96\%, $1.41 \mathrm{~T}$ (c) isostatic pressing $97 \%, 1.42 \mathrm{~T}$ (d) grain size distribution.
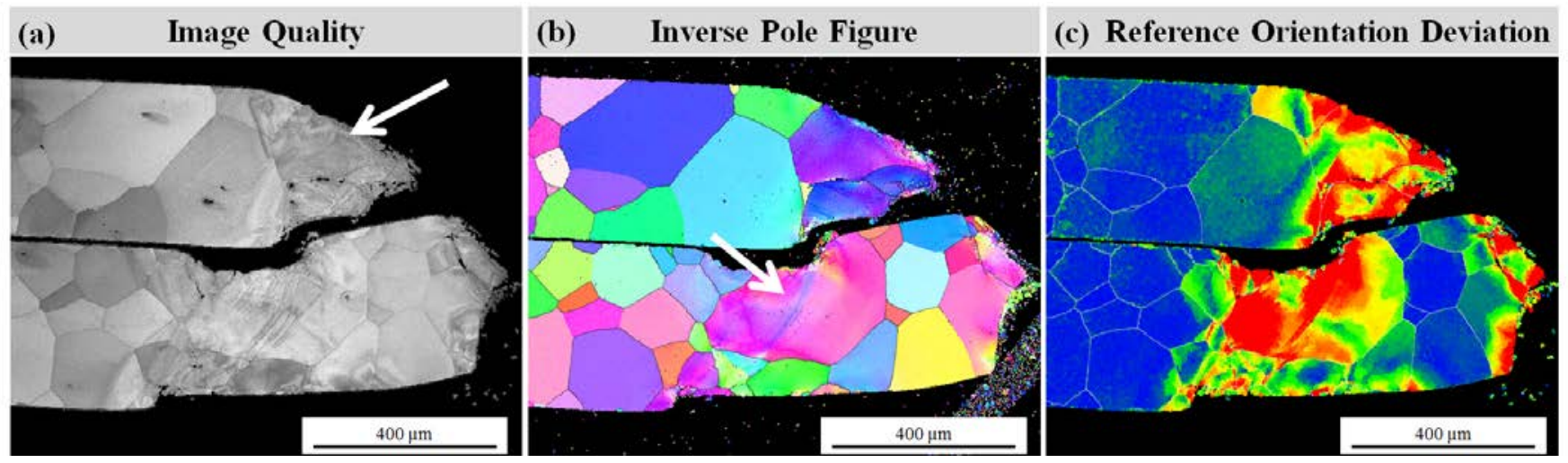

Figure 2. Three different types of EBSD information. The image quality (IQ) shows, that the signal intensity of the pattern decreases at the cutting edge. The inverse pole figure (IPF) illustrates the orientation deviation in deformed grains (see arrow). Additionally, the reference orientation deviation (ROD) allows the quantification of misorientations (blue: $<1^{\circ}$, red $\geq 10^{\circ}$ ). 